\title{
Euroroundup
}

\section{INFLUENZA ANTIVIRAL SUSCEPTIBILITY MONITORING ACTIVITIES IN RELATION TO NATIONAL ANTIVIRAL STOCKPILES IN EUROPE DURING THE WINTER $2006 / 2007$ SEASON}

\author{
A Meijer (a.meijer@nivel.nl) ${ }^{12}$, A Lackenby ${ }^{34}$, A Hay $^{35}$, M Zambon $^{34}$ \\ 1. European Influenza Surveillance Scheme (EISS) Co-ordination Centre, Nederlands Instituut voor Onderzoek van de Gezondheidszorg \\ (Netherlands Institute for Health Services Research, NIVEL), Utrecht, the Netherlands \\ 2. Laboratory for Infectious Diseases and Screening, Rijksinstituut voor Volksgezondheid en Milieu (National Institute of Public Health and the \\ Environment, RIVM), Bilthoven, the Netherlands \\ 3. European Surveillance Network for Vigilance against Viral Resistance (VIRGIL) \\ 4. Respiratory Virus Unit, Centre for Infection, Health Protection Agency, London, United Kingdom \\ 5. World Health Organization Collaborating Centre for Reference and Research on Influenza, National Institute for Medical Research, London, \\ United Kingdom
}

Due to the influenza pandemic threat, many countries are stockpiling antivirals in the hope of limiting the impact of a future pandemic virus. Since resistance to antiviral drugs would probably significantly alter the effectiveness of antivirals, surveillance programmes to monitor the emergence of resistance are of considerable importance. During the 2006/2007 influenza season, an inventory was conducted by the European Surveillance Network for Vigilance against Viral Resistance (VIRGIL) in collaboration with the European Influenza Surveillance Scheme (EISS) to evaluate antiviral susceptibility testing by the National Influenza Reference Laboratories (NIRL) in relation to the national antiviral stockpile in 30 European countries that are members of EISS. All countries except Ukraine had a stockpile of the neuraminidase inhibitor (NAI) oseltamivir. Additionally, four countries had a stockpile of the NAI zanamivir and three of the M2 ion channel inhibitor rimantadine. Of 29 countries with a NAI stockpile, six countries' NIRLs could determine virus susceptibility by $50 \%$ inhibitory concentration (IC50) and in 13 countries it could be done by sequencing. Only in one of the three countries with a rimantadine stockpile could the NIRL determine virus susceptibility, by sequencing only. However, including the 18 countries that had plans to introduce or extend antiviral susceptibility testing, the NIRLs of 21 of the 29 countries with a stockpile would be capable of susceptibility testing appropriate to the stockpiled drug by the end of the 2007/2008 influenza season. Although most European countries in this study have stockpiles of influenza antivirals, susceptibility surveillance capability by the NIRLs appropriate to the stockpiled antivirals is limited.

\section{Introduction}

The continued circulation and spread of highly pathogenic avian influenza virus $\mathrm{A}(\mathrm{H} 5 \mathrm{~N} 1)$ throughout large parts of Asia and several countries in Europe and Africa underlines the potential of animal (avian) reservoirs to act as the source of the next pandemic of influenza [1]. Since 2003, by 11 April 2007, 291 human cases with a case fatality rate of approximately $60 \%$ have been reported by the WHO [2]. Lack of human-to-human transmission has limited the burden of influenza $A(\mathrm{H} 5 \mathrm{~N} 1)$ virus as a human pathogen. However, given the genetic flexibility of influenza viruses, and experience of human morbidity and mortality following previous pandemics, developing counter measures is an important action to minimise the impact of a future pandemic. Many countries in Europe have already stockpiled antivirals as part of their pandemic preparedness plans. The ways in which national stockpiles would be used may vary substantially between countries, according to which drugs are stockpiled and how countries plan to use their resources at different stages of a pandemic [3].

Current licensed antivirals for therapy and/or prophylaxis of influenza fall into two classes, the adamantanes (amantadine and rimantadine), M2 ion channel inhibitors effective against influenza A viruses only, and the neuraminidase inhibitors (NAI) oseltamivir and zanamivir that are effective against both influenza $A$ and $B$ viruses.

During therapy with adamantanes, influenza viruses rapidly become resistant and are readily transmitted [4]. In addition, in many parts of the world, circulating $\mathrm{A}(\mathrm{H} 3 \mathrm{~N} 2)$ viruses have become naturally resistant [4]. In Cambodia, Vietnam and other parts of south-east Asia, a high proportion of $\mathrm{A}(\mathrm{H} 5 \mathrm{~N} 1)$ viruses isolated from poultry are adamantane resistant, and although resistance has been detailed in clade $1 \mathrm{~A}(\mathrm{H} 5 \mathrm{~N} 1)$ viruses, this is not uniform through all lineages of $A(H 5 N 1)[5,6]$. More recent clade $2 A(H 5 N 1)$ viruses have remained adamantane sensitive $[5,6]$.

For the NAI drugs, emergence of resistance to seasonal influenza viruses $[A(H 3 N 2), A(H 1 N 1)$ and $B]$ has been documented, with the highest frequency (18\%) being reported in children following oseltamivir treatment in Japan $[7,8]$. Oseltamivir resistance in severe human $\mathrm{A}(\mathrm{H} 5 \mathrm{~N} 1)$ virus infections has also been reported following therapy $[8,9]$. However, wide circulation of NAI resistant human strains has not been reported, although these can be detected at very low frequency in seasonal influenza surveillance programmes in countries with high drug consumption [10]. Currently, the ease of transmission of NAl resistant viruses is much less than that of adamantane resistant viruses, mainly because NAI resistance mutations tend to impair virus fitness and such viruses generally show reduced transmissibility in models in vivo [11].

In this context, VIRGIL and EISS have been collaborating since 2004 to develop capability and capacity for surveillance of influenza antiviral resistance within Europe [12,13]. VIRGIL (www.virgil-net. 
org), is an EU funded network of excellence that integrates and coordinates the activities of physicians and scientists from many institutions in 14 European countries in order to combat current and emerging antiviral drug resistance developments of influenza virus, Hepatitis B virus and Hepatitis C virus. For influenza, this includes the creation of reference laboratories for antiviral susceptibility measurement, development of testing protocols for phenotypic and genotypic analysis, training for laboratory staff and linkage of susceptibility testing to clinical surveillance activities. EISS (http://www.eiss.org), is a collaborative network of primary care physicians, epidemiologists and virologists that aims to contribute to a reduction in morbidity and mortality due to influenza in Europe by active clinical and virological surveillance of influenza, and has been operational since $1996[14,15]$. EISS is currently funded by the European Centre for Disease Prevention and Control (ECDC) and includes 26 European Union countries plus Norway, Serbia, Switzerland and Ukraine (Table 1,2). A total of 35 National Influenza Centres (NIC) recognised by the WHO plus five Influenza Reference Laboratories (IRL) in countries that have currently no NIC (Cyprus, Estonia, Lithuania, Malta and Ukraine), together referred to as NIRL for this paper, participate in EISS (http://www.eiss. org/cgi-files/wiw_cnrl_labs.cgi). These laboratories have functioned within EISS as the Community Network of Reference Laboratories for Human Influenza in Europe (CNRL) since 2003 [12,13]. The NIRLs report virus detection and identification data of respiratory specimens collected by sentinel physicians and of non-sentinel (e.g. hospital or non-NIRL laboratories) sources to EISS and work on improving the virological surveillance, which includes antiviral resistance monitoring.

Since the widespread emergence of antiviral resistance would likely have a significant impact on clinical effectiveness of antiviral therapy and prophylaxis, it is important to track resistance through regional and national surveillance programmes. In addition, it is important that these surveillance programmes are appropriate to the antivirals being stockpiled. Therefore, VIRGIL and EISS, as the European public-funded consortia working on these subjects, carried out this study in order to evaluate the actual and future antiviral susceptibility testing activities by the NIRLs in relation to the national antiviral stockpile in the 30 European countries that are members of EISS.

\section{Method}

During a VIRGIL-EISS laboratory workshop on influenza antiviral susceptibility testing techniques at the Health Protection Agency (HPA) in London between 3 and 6 October 2006, the participants who represented 17 NIRLs were asked the following questions:

1. Does your country have a stockpile of influenza antiviral drugs?

2. If yes, which antiviral drugs?

3. Do you do any NAI susceptibility testing?

4. If yes, which tests: genotypic (virus nucleic acid analysis) or phenotypic (virus susceptibility analysis, i.e. determination of $50 \%$ inhibitory concentration values)?

5 . Do you do any adamantane susceptibility testing?

6. If yes, which tests (genotypic/phenotypic)?

7. Do you plan to either introduce or extend NAI or adamantane susceptibility testing in the season 2006/2007?

The questionnaire was also sent out more widely after the course by the EISS coordination centre in the period October
2006 - January 2007 to all 40 EISS NIRLs for verification and for completion by the remaining 23 NIRLs.

Answers to the questionnaire were processed at the EISS coordination centre and reports were created by laboratory and by country. When a country had more than one NIRL the most positive answer was taken for the report by country. If the answer to a question was: "available", "if necessary" or "possibly", these were interpreted in the analysis as no actual testing or no actual plans to introduce or extend testing.

\section{Results}

At least one NIRL per country responded to the inventory questionnaire. In total, 33 of the 40 NIRLs in EISS responded. The London NIC reported on behalf of the United Kingdom, which includes also the NICs in Northern Ireland, Scotland and Wales. Of those 33 NIRLs one NIRL did not provide information on sequencing of the neuraminidase gene and 17 NIRLs did not report on actual phenotypic testing for adamantane susceptibility. Tables 1 and 2 summarise the results.

Out of 33 NIRLs representing 30 countries, seven NIRLs (six countries) reported phenotypic testing of NAI susceptibility and 15 NIRLs (13 countries) reported sequencing of the neuraminidase gene. Sixteen NIRLs (16 countries) reported on the capacity for phenotypic testing of adamantane susceptibility, of which only one was testing. Out of 33 NIRLs (30 countries), seven NIRLs (seven countries) reported sequencing of the M2 gene.

All 30 EISS countries apart from Ukraine had a stockpile of antivirals; 22 countries had stockpiled oseltamivir only, four countries had stockpiled both oseltamivir and zanamivir and three oseltamivir and rimantadine. In six out of the 29 countries that had a stockpile of NAI drugs (oseltamivir or zanamivir), the NIRLs were performing the phenotypic analysis to evaluate NAI resistance, and in 13 out of the 29 countries with a stockpile of NAI drugs the NIRLs undertook genotypic analysis of NA genes to assess NAI resistance. For the monitoring of adamantane resistance, only the NIRL of one out of the three countries with a stockpile of rimantadine indicated actual testing of adamantane susceptibility, by genotypic means only.

In 21 of the 30 countries, NIRLs had plans to introduce or extend antiviral susceptibility testing for NAls and/or adamantanes over the next winter seasons using either phenotypic analysis (17 NIRLs in 14 countries for NAls and 15 NIRLs in 13 countries for adamantanes) and/or genotypic analysis (22 NIRLs in 20 countries for NAIs and 16 NIRLs in 14 countries for adamantanes), while NIRLs in nine countries had no plans to introduce any testing. Taking into account these planned activities, NIRLs in 14 out of the 29 countries with a NAI stockpile would have the capacity to perform the phenotypic analysis, and NIRLs in 20 countries would have the capacity to undertake genotypic analysis to assess NAI resistance. For the monitoring of adamantane resistance, the NIRLs in two out of the three countries with a stockpile of rimantadine would have the capacity to conduct adamantane susceptibility analysis, by phenotypic and/or genotypic means. 
TA B L E 1

Overview of influenza antiviral susceptibility monitoring activities in Europe during the 2006/2007 influenza season

\begin{tabular}{|c|c|c|c|c|c|c|c|c|c|c|c|c|c|c|}
\hline \multirow{4}{*}{ Country $(\mathrm{N}=30)$} & \multirow{4}{*}{$\begin{array}{c}\text { National } \\
\text { stockpile } \\
\text { of antiviral } \\
\text { drug }\end{array}$} & \multirow{4}{*}{ Laboratory(N=33) } & \multicolumn{12}{|c|}{ Current laboratory antiviral susceptibility activities } \\
\hline & & & \multicolumn{6}{|c|}{ Neuraminidase inhibitor susceptibility } & \multicolumn{6}{|c|}{ Adamantane susceptibility } \\
\hline & & & \multicolumn{3}{|c|}{ Phenotypic testing ${ }^{1}$} & \multicolumn{3}{|c|}{ Sequencing NA gene } & \multicolumn{3}{|c|}{ Phenotypic testing ${ }^{1}$} & \multicolumn{3}{|c|}{ Sequencing $\mathrm{M} 2$ gene } \\
\hline & & & Yes & No & Remark & Yes & No & Remark & Yes & No & Remark & Yes & No & Remark \\
\hline Austria & Oseltamivir & NIC, Vienna & & $x$ & & $x$ & & & & $x$ & & & $x$ & \\
\hline Belgium & Oseltamivir & NIC, Brussels & & $x$ & & & $x$ & & & $x$ & & & $x$ & \\
\hline Cyprus & $\begin{array}{l}\text { Oseltamivir } \\
+ \text { Zanamivir } \\
\end{array}$ & IRL, Nicosia & & $x$ & & & $x$ & & & $x$ & & & $x$ & \\
\hline Czech Republic & Oseltamivir & NIC, Prague & & $x$ & & & $x$ & & & & Available & & $x$ & \\
\hline Denmark & Oseltamivir & NIC, Copenhagen & $x$ & & & $x$ & & & & & Missing & $x$ & & \\
\hline Estonia & Oseltamivir & IRL, Tallinn & & $x$ & & & $x$ & & & & Missing & & $x$ & \\
\hline Finland & Oseltamivir & NIC, Helsinki & & $x$ & & $x$ & & & & & Available & & $x$ & \\
\hline \multirow[t]{2}{*}{ France } & Oseltamivir & NIC, Paris & $x$ & & & $x$ & & & & & $\begin{array}{c}\text { If } \\
\text { necessary }\end{array}$ & $x$ & & \\
\hline & & NIC, Lyon & $x$ & & & $x$ & & & & & Missing & & $x$ & \\
\hline Germany & \begin{tabular}{|} 
Oseltamivir \\
+ Zanamivir
\end{tabular} & NIC, Berlin & & $x$ & & $x$ & & & & & Missing & & $x$ & \\
\hline \multirow[t]{2}{*}{ Greece } & Oseltamivir & NIC, Thessaloniki & & $x$ & & & $x$ & & & & Missing & & $x$ & \\
\hline & & NIC, Athens & & $x$ & & & $x$ & & & & Missing & & $x$ & \\
\hline Hungary & Oseltamivir & NIC, Budapest & $x$ & & & $x$ & & & & $x$ & & & $x$ & \\
\hline Ireland & Oseltamivir & NIC, Dublin & & $x$ & & & $x$ & & & $x$ & & & $x$ & \\
\hline \multirow[t]{2}{*}{ Italy } & $\begin{array}{l}\text { Oseltamivir } \\
+ \text { Zanamivir } \\
\end{array}$ & NIC, Rome & & $x$ & & $x$ & & & & & Missing & & $x$ & \\
\hline & & NIC, Milan & & $x$ & & $x$ & & & & & Missing & & $x$ & \\
\hline Latvia & $\begin{array}{c}\text { Oseltamivir } \\
+ \\
\text { Rimantadine }\end{array}$ & NIC, Riga & & $x$ & & & $x$ & & & & Missing & & $x$ & \\
\hline Lithuania & $\begin{array}{c}\text { Oseltamivir } \\
+ \\
\text { Rimantadine }\end{array}$ & IRL, Vilnius & & $x$ & & & & Missing & & & Missing & & & Possibly \\
\hline Luxembourg & Oseltamivir & NIC, Luxembourg & & $x$ & & & $x$ & & & $x$ & & & $x$ & \\
\hline Malta & Oseltamivir & IRL, Malta & & $x$ & & & $x$ & & & $x$ & & & $x$ & \\
\hline \begin{tabular}{|l} 
Netherlands \\
\end{tabular} & Oseltamivir & NIC/RIVM, Bilthoven & $x$ & & & $x$ & & & $x$ & & & $x$ & & \\
\hline Norway & \begin{tabular}{|c|} 
Oseltamivir \\
+ \\
Rimantadine
\end{tabular} & NIC, Oslo & & $x$ & & $x$ & & & & $x$ & & $x$ & & \\
\hline Poland & Oseltamivir & NIC, Warsaw & & $x$ & & & $x$ & & & $x$ & & & $x$ & \\
\hline Portugal & Oseltamivir & NIC, Lisbon & & $x$ & & $x$ & & & & & Missing & & $x$ & \\
\hline Romania & Oseltamivir & NIC, Bucharest & & $x$ & & & $x$ & & & $x$ & & & $x$ & \\
\hline Serbia & Oseltamivir & NIC, Belgrade & & $x$ & & $x$ & & & & & Missing & & $x$ & \\
\hline Slovakia & Oseltamivir & NIC, Bratislava & & $x$ & & & $x$ & & & & Missing & & $x$ & \\
\hline Slovenia & Oseltamivir & NIC, Ljubljana & & $x$ & & & $x$ & & & $x$ & & & $x$ & \\
\hline Spain & Oseltamivir & NIC, Madrid & & $x$ & & & $x$ & & & & Missing & & $x$ & \\
\hline Sweden & Oseltamivir & NIC, Stockholm & $x$ & & & $x$ & & & & & Missing & $x$ & & \\
\hline Switzerland & Oseltamivir & NIC, Geneva & & $x$ & & & $x$ & & & & Missing & $x$ & & \\
\hline \multirow[t]{2}{*}{ Ukraine } & Planned & IRL, Kiev & & $x$ & & & $x$ & & & $x$ & & & $x$ & \\
\hline & \begin{tabular}{|l|} 
Rimantadine \\
- in private \\
pharmacies
\end{tabular} & & & & & & & & & & & & & \\
\hline \begin{tabular}{|c|} 
United \\
Kingdom \\
\end{tabular} & $\begin{array}{l}\text { Oseltamivir } \\
+ \text { Zanamivir }\end{array}$ & NIC, London & $x$ & & Limited $^{2}$ & $x^{2}$ & & & & & Missing & $x$ & & \\
\hline \begin{tabular}{|c|} 
Ringaom \\
Total \\
laboratories
\end{tabular} & & & 7 & 26 & & 15 & 17 & & 1 & 12 & & 7 & 25 & \\
\hline \begin{tabular}{c|}
$\begin{array}{c}\text { Total } \\
\text { countries }\end{array}$ \\
\end{tabular} & & & 6 & 24 & & 13 & 16 & & 1 & 12 & & 7 & 23 & \\
\hline
\end{tabular}

1 Phenotypic = IC50 determination using fluorescent assay (MUNANA) for NAI, or Cell ELISA or plaque inhibition assay for NAI and adamantanes 2 As part of the VIRGIL project 
T A B L E $\mathbf{2}$

Overview of influenza antiviral susceptibility monitoring activities planned to be introduced or extended during the $2006 / 2007$ influenza season

\begin{tabular}{|c|c|c|c|c|c|c|c|c|c|c|c|c|c|c|}
\hline \multirow{4}{*}{ Country $(N=30)$} & \multirow{4}{*}{$\begin{array}{l}\text { National stockpile of } \\
\text { antiviral drug }\end{array}$} & \multirow{4}{*}{ Laboratory(N=33) } & \multicolumn{12}{|c|}{ Planned laboratory antiviral susceptibility activities } \\
\hline & & & \multicolumn{6}{|c|}{$\begin{array}{c}\text { Neuraminidase inhibitor } \\
\text { susceptibility }\end{array}$} & \multicolumn{6}{|c|}{ Adamantane susceptibility } \\
\hline & & & \multicolumn{3}{|c|}{$\begin{array}{l}\text { Phenotypic } \\
\text { testing }\end{array}$} & \multicolumn{3}{|c|}{$\begin{array}{c}\begin{array}{c}\text { Sequencing NA } \\
\text { gene }\end{array} \\
\end{array}$} & \multicolumn{3}{|c|}{ Phenotypic testing ${ }^{1}$} & \multicolumn{3}{|c|}{ Sequencing M2 gene } \\
\hline & & & Yes & No & Remark & Yes & No & Remark & Yes & No & Remark & Yes & No & Remark \\
\hline Austria & Oseltamivir & NIC, Vienna & & & Possibly & $x$ & & & & $x$ & & & $x$ & \\
\hline Belgium & Oseltamivir & NIC, Brussels & & $x$ & & & $x$ & & & $x$ & & & $x$ & \\
\hline Cyprus & Oseltamivir + Zanamivir & IRL, Nicosia & & $x$ & & & $x$ & & & $x$ & & & $x$ & \\
\hline Czech Republic & Oseltamivir & NIC, Prague & $x$ & & & $x$ & & & $x$ & & & $x$ & & \\
\hline Denmark & Oseltamivir & NIC, Copenhagen & $x$ & & & $x$ & & & $x$ & & & $x$ & & \\
\hline Estonia & Oseltamivir & IRL, Tallinn & & & Possibly & $x$ & & & & & Possibly & & & Possibly \\
\hline Finland & Oseltamivir & NIC, Helsinki & $x$ & & & $x$ & & & $x$ & & & $x$ & & \\
\hline \multirow[t]{2}{*}{ France } & Oseltamivir & NIC, Paris & $x$ & & & $x$ & & & $x$ & & & $x$ & & \\
\hline & & NIC, Lyon & $x$ & & & $x$ & & & $x$ & & & $x$ & & \\
\hline Germany & Oseltamivir + Zanamivir & NIC, Berlin & $x$ & & & $x$ & & & & & If necessary & & & If necessary \\
\hline \multirow[t]{2}{*}{ Greece } & Oseltamivir & NIC, Thessaloniki & $x$ & & & $x$ & & & & $x$ & & $x$ & & \\
\hline & & NIC, Athens & $x$ & & & & & Possibly & & $x$ & & & $x$ & \\
\hline Hungary & Oseltamivir & NIC, Budapest & $x$ & & & $x$ & & & $x$ & & & $x$ & & \\
\hline Ireland & Oseltamivir & NIC, Dublin & & $x$ & & $x$ & & & & $x$ & & & $x$ & \\
\hline \multirow[t]{2}{*}{ Italy } & Oseltamivir + Zanamivir & NIC, Rome & $x$ & & & $x$ & & & $x$ & & & $x$ & & \\
\hline & & NIC, Milan & $x$ & & & $x$ & & & $x$ & & & $x$ & & \\
\hline Latvia & $\begin{array}{l}\text { Oseltamivir + } \\
\text { Rimantadine }\end{array}$ & NIC, Riga & $x$ & & & & $x$ & & $x$ & & & & $x$ & \\
\hline Lithuania & $\begin{array}{l}\text { Oseltamivir + } \\
\text { Rimantadine } \\
\end{array}$ & IRL, Vilnius & & & Possibly & & & Possibly & & $x$ & & & & Possibly \\
\hline Luxembourg & Oseltamivir & NIC, Luxembourg & & $x$ & & $x$ & & & & $x$ & & & $x$ & \\
\hline Malta & Oseltamivir & IRL, Malta & & $x$ & & & $x$ & & & $x$ & & & $x$ & \\
\hline Netherlands & Oseltamivir & NIC/RIVM, Bilthoven & $x$ & & & $x$ & & & $x$ & & & $x$ & & \\
\hline Norway & $\begin{array}{l}\text { Oseltamivir + } \\
\text { Rimantadine }\end{array}$ & NIC, Oslo & & & Possibly & $x$ & & & $x$ & & & $x$ & & \\
\hline Poland & Oseltamivir & NIC, Warsaw & & $x$ & & & $x$ & & & $x$ & & & $x$ & \\
\hline Portugal & Oseltamivir & NIC, Lisbon & $x$ & & & $x$ & & & $x$ & & & $x$ & & \\
\hline Romania & Oseltamivir & NIC, Bucharest & & & Possibly & & & Possibly & & $x$ & & & $x$ & \\
\hline Serbia & Oseltamivir & NIC, Belgrade & $x$ & & & $x$ & & & & $x$ & & & $x$ & \\
\hline Slovakia & Oseltamivir & NIC, Bratislava & & & Possibly & & $x$ & & & $x$ & & & $x$ & \\
\hline Slovenia & Oseltamivir & NIC, Ljubljana & & $x$ & & & $x$ & & & $x$ & & & $x$ & \\
\hline Spain & Oseltamivir & NIC, Madrid & & $x$ & & $x$ & & & $x$ & & & $x$ & & \\
\hline Sweden & Oseltamivir & NIC, Stockholm & $x$ & & & $x$ & & & $x$ & & & $x$ & & \\
\hline Switzerland & Oseltamivir & NIC, Geneva & & $x$ & & $x$ & & & $x$ & & & $x$ & & \\
\hline \multirow[t]{2}{*}{ Ukraine } & Planned & IRL, Kiev & & $x$ & & & $x$ & & & $x$ & & & $x$ & \\
\hline & $\begin{array}{c}\text { Rimantadine - in private } \\
\text { pharmacies }\end{array}$ & & & & & & & & & & & & & \\
\hline United Kingdom & Oseltamivir + Zanamivir & NIC, London & $x$ & & & $x^{2}$ & & & & $x$ & & $x$ & & \\
\hline Total laboratories & & & 17 & 10 & & 22 & 8 & & 15 & 16 & & 16 & 14 & \\
\hline Total countries & & & 14 & 10 & & 20 & 8 & & 13 & 15 & & 14 & 14 & \\
\hline
\end{tabular}

1 Phenotypic = IC50 determination using fluorescent assay (MUNANA) for NAI, or Cell ELISA or plaque inhibition assay for NAI and adamantanes 2 Pyrosequencing, as part of the VIRGIL project 


\section{Discussion}

All European countries (except Ukraine) that participated in this study indicated that antiviral stockpiles are available. This is an encouraging observation as it relates directly to national pandemic preparedness activities. However, the number of countries in which NIRLs performed influenza antiviral susceptibility testing appropriate to the stockpiled antivirals was limited.

There is widespread variation in the use of different influenza antiviral drugs within Europe (Intercontinental Marketing Services prescribing data, Oct 2006), and substantial variation in natural susceptibility of circulating influenza strains to adamantane class of drugs in particular [4]. The creation of antiviral stockpiles involves substantial allocation of resources, and in the event of a pandemic it will be important to use such resources efficiently. Emerging information about the potential for the development of resistance against influenza antiviral drugs suggests that information about susceptibility of circulating strains should be taken into consideration for decisions on recommending drug use, in particular in those countries which have stockpiles of adamantane drugs, where the level of resistance is high, but not uniform, among circulating strains [4]. Antiviral susceptibility testing should exist to support the stockpiling of antiviral drugs, to analyse the susceptibility of viruses to the stockpiled antivirals in the early stages of a pandemic and to assess possible treatment failure.

European antiviral susceptibility monitoring is currently achieved through the VIRGIL project, which will finish early in 2008. Representative, but limited, evaluation of both NAI and adamantane resistance of European influenza isolates is carried out in London by the HPA in collaboration with the World Health Organization Collaborating Centre for Reference and Research on Influenza (WHO-CC) at Mill Hill. Subsets of isolates from all European countries that undergo detailed analyses for the annual WHO vaccine recommendations have been analysed, representing approximately $5-10 \%$ of virus isolates in Europe each season. Aggregated susceptibility data for winter seasons 2004/2005 and 2005/2006 will shortly be available through the EISS/VIRGIL website and analysis of isolates from the $2006 / 2007$ season is underway.

As the VIRGIL project is limited in time, national testing should be developed. This should be done as a matter of urgency, as our inventory showed that NIRLs in only 13 out of 29 countries with a NAI stockpile were testing for NAI susceptibility. In addition, the NIRL in only one out of three countries with a stockpile of rimantadine was testing for adamantane susceptibility. Therefore, VIRGIL in collaboration with EISS is working on improving this situation. As the NIRLs in EISS process the respiratory specimens collected by sentinel doctors, of whom the patient lists are representative for the population in a country, this existing network offers an excellent opportunity for setting up antiviral susceptibility surveillance programmes $[14,15]$.

Most NIRLs test for NAI resistance by sequencing, indicating that this type of analysis is much more widespread, compared with phenotypic susceptibility testing that is dependent on working with virus isolates. However, phenotypic NAI susceptibility analysis is still necessary to fully evaluate NAI resistance, given the uncertainty of purely genotypic methods for assessment of resistance as only a few mutations conferring NAI resistance have been described so far, and several more are likely to emerge. Therefore, we recommend that if a surveillance programme for NAls is developed, both genotypic and phenotypic methods are used and data combined from both methods. In contrast, if a surveillance programme for adamantane susceptibility is developed, testing using genotypic methods would be sufficient as the relationship between genotype and phenotype is absolutely predictable so that either method for analysis is suitable. In addition, it will be necessary to ensure that capability for drug resistance surveillance is maintained, testing methodologies and data are shared and harmonised between countries as surveillance for drug susceptibility is expanded.

To facilitate the development of above-mentioned surveillance programmes the following actions have been taken:

- A swabbing protocol has been published in the EISS library that includes a form asking for a minimum set of data i.e. questions about antiviral treatment/prophylaxis of the patient, the kind of drugs that have been used and contacts with family members with flu who used antiviral drugs.

- Standard operating procedures for phenotypic and genotypic analysis of antiviral susceptibility have been published in the EISS laboratory protocol library.

- A laboratory training programme hosted by VIRGIL, the HPA and the WHO-CC, National Institute for Medical Research, London, was held in October 2006 to assist national laboratories in developing their capacity for techniques involved in antiviral susceptibility. This meeting was attended by representatives from NIRLs in 17 countries.

In addition to these activities, our inventory showed that several countries were planning to initiate or extend antiviral surveillance over the next winter seasons. All together, these are the first steps to enhancing capacity and capability for detection of influenza antiviral resistance in Europe.

\section{Conclusion}

Although stockpiles of influenza antivirals are available in almost all EISS countries in Europe, surveillance systems to track antiviral resistance necessary to support the use of the stockpiled drugs are not widely available. Through collaborative efforts of VIRGIL and EISS, countries are being facilitated to develop antiviral susceptibility surveillance systems. This will further strengthen the level of pandemic preparedness in Europe as enhancing antiviral susceptibility monitoring capacity and capability will improve ability to deliver rapid information on the appropriateness of using the stockpiled antivirals in case of an introduction of a new, possible pandemic, influenza A virus subtype.

\section{Acknowledgements}

The authors thank all colleagues from the participating laboratories, VIRGIL and EISS who provided information.

\section{References}

1. World Organisation for Animal Health (OIE). Update on avian influenza in animals (type H5). Available from: http://www.oie.int/downld/AVIAN\%2OINFLUENZA/A_AIAsia.htm

2. World Health Organization (WHO). Cumulative number of confirmed human cases of avian influenza A/(H5N1) reported to WHO. Available from: http://www.who. int/csr/disease/avian_influenza/country/cases_table_2007_04_11/en/index. html

3. Mounier-Jack S, Coker R. How prepared is Europe for Pandemic Influenza? An Analysis of National Plans. Available from: http://www.lshtm.ac.uk/ecohost/ projects/pandemic/HowpreparedisEurope.pdf

4. Bright RA, Medina MJ, Xu X, Perez-Oronoz G, Wallis TR, Davis XM, et al. 
Incidence of adamantane resistance among influenza A (H3N2) viruses isolated worldwide from 1994 to 2005: a cause for concern. Lancet. 2005;366:117581.

5. Cheung CL, Rayner JM, Smith GJ, Wang P, Naipospos TS, Zhang J, et al. Distribution of amantadine-resistant $\mathrm{H} 5 \mathrm{~N} 1$ avian influenza variants in Asia. J Infect Dis. 2006;193:1626-9.

6. World Health Organization Global Influenza Program Surveillance Network. Evolution of H5N1 avian influenza viruses in Asia. Emerg Infect Dis. 2005;11:1515-21.

7. Kiso M, Mitamura K, Sakai-Tagawa Y, Shiraishi K, Kawakami C, Kimura K, et al. Resistant influenza A viruses in children treated with oseltamivir: descriptive study. Lancet. 2004;364:759-65.

8. Moscona A. Oseltamivir resistance--disabling our influenza defenses. N Engl J Med. 2005;353:2633-6.

9. Le QM, Kiso M, Someya K, Sakai YT, Nguyen TH, Nguyen KH, et al. Avian flu: isolation of drug-resistant H5N1 virus. Nature. 2005;437:1108.

10. Monto AS, McKimm-Breschkin JL, Macken C, Hampson AW, Hay A, Klimov A, et al. Detection of influenza viruses resistant to neuraminidase inhibitors in global surveillance during the first 3 years of their use. Antimicrob Agents global surveillance during the
11. Yen HL, Herlocher LM, Hoffmann E, Matrosovich MN, Monto AS, Webster RG, et al. Neuraminidase inhibitor-resistant influenza viruses may differ substantially in fitness and transmissibility. Antimicrob Agents Chemother. 2005;49:4075-

12. Meijer A, Valette M, Manuguerra JC, Perez-Brena P, Paget J, Brown C, et al. Implementation of the community network of reference laboratories for human influenza in Europe. J Clin Virol. 2005;34:87-96.

13. Meijer A, Brown C, Hungnes 0 , Schweiger B, Valette M, van der Werf S, et al. Programme of the community network of reference laboratories for human influenza to improve influenza surveillance in Europe. Vaccine. 2006;24:671723.

14. Aymard M, Valette $M$, Lina $B$, Thouvenot $D$, the members of Groupe Régional d'Observation de la Grippe and European Influenza Surveillance Scheme. Surveillance and impact of influenza in Europe. Vaccine. 1999:17:S30-S41.

15. Fleming DM, van der Velden J, Paget WJ. The evolution of influenza surveillance in Europe and prospects for the next ten years. Vaccine. 2003; 21:1749-53.

Citation style for this article: Meijer A, Lackenby A, Hay A, Zambon M, . Influenza antiviral susceptibility monitoring activities in relation to national antiviral stockpiles in Europe during the winter 2006/2007 season. Euro Surveill 2007;12(4)[Epub ahead of print]. Available online: http://www.eurosurveillance.org/em/v12n04/1204-222.asp 